\title{
Relationships between Changes in Urban Characteristics and Air Quality in East Asia from 2000 to 2010
}

\author{
Andrew Larkin*, Aaron van Donkelaar, Jeffrey A. Geddes, Randall V. Martin, Perry Hystad \\ *Corresponding email: Andrew.Larkin@oregonstate.edu \\ *Corresponding telephone: 001-541-737-2743
}

Supplemental tables include a list of data sources and saturated regression models. Supplemental figures include variance-covariance matrices, compactness index examples, and maps of spatial distributions of several key varaibles. 
Table S1. Data sources and characteristics

\begin{tabular}{|l|lll|}
\hline & Source & $\begin{array}{l}\text { Spatial } \\
\text { Resolution }\end{array}$ & Units \\
\hline $\mathbf{P M}_{2.5}$ & Van Donkelaar et al. $^{14}$ & $0.1^{\circ}$ & $\mu \mathrm{g} / \mathrm{m}^{3}$ \\
$\mathbf{N O}_{2}$ & Lamsal et al. $^{13}$ & $0.1^{\circ}$ & $\mathrm{ppb}$ \\
Urban Classification & World Bank & $250 \mathrm{~m}$ & categorical $^{\circ}$ \\
Population Density & NASA SEDAC & $1 \mathrm{~km}$ & persons* $^{*} \mathrm{~km}^{-1}$ \\
NDVI & NASA LP DAAC & $250 \mathrm{~m}$ & Relative scale $(-1-1)$ \\
Impervious Surface Area & NOAA NGDC & $1 \mathrm{~km}$ & $\%$ \\
Light at Night & NOAA NGDC & $30^{\prime \prime}$ & $\%$ \\
Rainfall & NASA EOC & $0.25^{\circ}$ & $\mathrm{mm}$ \\
Temperature & NASA EOC & $0.1^{\circ}$ & ${ }^{\circ} \mathrm{C}$ \\
\hline
\end{tabular}

* primary and secondary roads and turnoffs ** tertiary and residential roads and turnoffs ***sum of major and minor density. 
Table S2. Non-stratified multivariate linear regression models for change in $\mathrm{NO}_{2}$ and $\mathrm{PM}_{2.5}$.

\begin{tabular}{|c|c|c|c|c|}
\hline & \multicolumn{2}{|c|}{$\mathrm{NO}_{2}$} & \multicolumn{2}{|c|}{$\mathbf{P M}_{2.5}$} \\
\hline & Coefficient & Std Error & Coefficient & Std Error \\
\hline \multicolumn{5}{|l|}{ Urban Expansion } \\
\hline Area (sq km) & $1.27 \times 10^{-3}$ & $2.18 \times 10^{-4} * * *$ & $4.99 \times 10^{-4}$ & $1.13 \times 10^{-3}$ \\
\hline Population (1000s) & $-2.42 \times 10^{-7}$ & $5.14 \times 10^{-7}$ & $-5.77 \times 10^{-7}$ & $2.65 \times 10^{-6}$ \\
\hline \multicolumn{5}{|c|}{ Change in Urban Characteristics } \\
\hline Compactness Index (0-1) & -2.31 & $1.10^{*}$ & -10.08 & 5.68 \\
\hline Population Density & $-1.75 \times 10^{-5}$ & $3.32 \times 10^{-6}$ & $-8.34 \times 10^{-7}$ & $1.71 \times 10^{-5}$ \\
\hline NDVI (-1-1) & 0.15 & $9.70 \times 10^{-2}$ & -0.49 & 0.50 \\
\hline Impervious Surface Area (\%) & -0.10 & $1.91 \times 10^{-2} * * *$ & -0.32 & $9.84 \times 10^{-2} * *$ \\
\hline Lights at Night (\%) & $7.99 \times 10^{-2}$ & $1.98 \times 10^{-2 * * *}$ & 0.31 & $0.10^{* *}$ \\
\hline \multicolumn{5}{|c|}{ Change in Urban Characteristic Heterogeneity } \\
\hline Population Density & $7.61 \times 10^{-5}$ & $2.74 \times 10^{-5^{* *}}$ & $6.13 \times 10^{-4}$ & $1.41 \times 10^{-4^{* * *}}$ \\
\hline NDVI (-1-1) & -0.15 & 0.28 & -1.54 & 1.46 \\
\hline Impervious Surface Area (\%) & $3.49 \times 10^{-2}$ & $2.06 \times 10^{-2}$ & 0.16 & 0.11 \\
\hline Lights at Night (\%) & -0.11 & $3.49 \times 10^{-2^{* *}}$ & -0.41 & $0.18^{*}$ \\
\hline \multicolumn{5}{|c|}{ Baseline (2000) Urban Characteristics } \\
\hline Area (sq km) & $1.45 \times 10^{-5}$ & $5.75 x^{-4}$ & $4.14 \times 10^{-3}$ & $3.00 x^{-3}$ \\
\hline Population (1000s) & $-6.17 \times 10^{-5}$ & $1.65 \times 10^{-4}$ & $-4.24 \times 10^{-4}$ & $8.50 \times 10^{-4}$ \\
\hline NDVI (-1-1) & $6.37 \times 10^{-3}$ & $5.55 \times 10^{-3}$ & $5.35 \times 10^{-2}$ & $2.87 \times 10^{-2}$ \\
\hline Impervious Surface Area (\%) & $-7.82 \times 10^{-2}$ & $1.40 \times 10^{-2} * * *$ & -0.187 & $7.25 \times 10^{-2} *$ \\
\hline Lights at Night (\%) & $4.01 \times 10^{-2}$ & $1.64 \times 10^{-2} *$ & $-9.04 \times 10^{-2}$ & $8.45 \times 10^{-2}$ \\
\hline \multicolumn{5}{|l|}{ Adjustment Factors } \\
\hline Distance to Coast $(\mathrm{km})$ & $-2.11 \times 10^{-3}$ & $2.762 \times 10^{-4 * * *}$ & $-7.78 \times 10^{-3}$ & $1.43 \times 10^{-3} * * *$ \\
\hline Annual Rainfall (mm) & $-4.88 \times 10^{-2}$ & $6.13 \times 10^{-3 * * *}$ & -0.16 & $3.17 \times 10^{-2} * * *$ \\
\hline Annual Temperature $\left({ }^{\circ} \mathrm{C}\right)$ & $-2.15 \times 10^{-2}$ & $6.56 \times 10^{-3} * *$ & -0.14 & $3.39 \times 10^{-2} * *$ \\
\hline Adjusted R ${ }^{2}$ & \multicolumn{2}{|c|}{0.46} & \multicolumn{2}{|r|}{0.34} \\
\hline
\end{tabular}

${ }^{*}$ p-value $<0.05, * *$ p-value $<0.01, * * *$ p-value $<0.001$ 
Table S3. Associations between $\mathrm{NO}_{2}$ change and urban expansion and urban characteristics - full model.

\begin{tabular}{|c|c|c|c|c|c|c|c|c|c|}
\hline & \multicolumn{9}{|c|}{ Urban Size } \\
\hline & \multicolumn{3}{|c|}{$100,000-250,000(n=235)$} & \multicolumn{3}{|c|}{$250,000-1,000,000(n=340)$} & \multicolumn{3}{|c|}{$>1,000,000(n=255)$} \\
\hline & Coefficient & Std Error & $\mathbf{R 2}$ & Coefficient & Std Error & R2 & Coefficient & Std Error & $\mathbf{R 2}$ \\
\hline \multicolumn{10}{|l|}{ Urban Expansion } \\
\hline Area (sq km) & $2.15 \times 10^{-2}$ & $7.88 \times 10^{-3} * *$ & 0.03 & $1.05 \times 10^{-2}$ & $2.96 \times 10^{-3} * * *$ & 0.08 & $1.22 \times 10^{-3}$ & $2.34 \times 10^{-4} * * *$ & 0.13 \\
\hline Population (1000s) & $5.04 \times 10^{-3}$ & $3.50 \times 10^{-3}$ & 0.08 & $-1.11 \times 10^{-3}$ & $9.91 \times 10^{-4}$ & 0.05 & $-3.81 \times 10^{-6}$ & $5.61 \times 10^{-6}$ & 0.02 \\
\hline $\mathrm{R} 2$ & & & 0.11 & & & 0.13 & & & 0.15 \\
\hline \multicolumn{10}{|c|}{ Change in Urban Characteristics } \\
\hline Compactness Index (0-1) & 2.42 & 1.99 & $<0.01$ & -2.34 & 1.87 & $<0.01$ & 3.68 & 2.39 & $<0.01$ \\
\hline Population Density & $-1.79 \times 10^{-5}$ & $1.58 \times 10^{-4}$ & $<0.01$ & $7.75 \times 10^{-5}$ & $6.18 \times 10^{-5}$ & 0.01 & $1.99 \times 10^{-6}$ & $3.59 \times 10^{-6}$ & 0.01 \\
\hline NDVI (-1-1) & -1.69 & 1.75 & 0.04 & 1.00 & 1.40 & 0.02 & $-1.13 \times 10^{-2}$ & 2.13 & 0.04 \\
\hline Impervious Surface Area (\%) & -0.16 & $3.96 \times 10^{-2} * * *$ & $<0.01$ & $-8.71 \times 10^{-2}$ & $2.68 \times 10^{-2} * *$ & 0.01 & $-8.14 \times 10^{-2}$ & $4.44 \times 10^{-2}$ & $<0.01$ \\
\hline Lights at Night (\%) & $9.96 \times 10^{-2}$ & $2.91 \times 10^{-2} * * *$ & 0.05 & $9.09 \times 10^{-2}$ & $3.41 \times 10^{-2} * *$ & 0.05 & 0.19 & $5.54 \times 10^{-2} * *$ & 0.25 \\
\hline R2 & & & 0.10 & & & 0.10 & & & 0.31 \\
\hline \multicolumn{10}{|c|}{ Change in Urban Characteristic Heterogeneity } \\
\hline Population Density & $-6.71 \times 10^{-5}$ & $5.19 \times 10^{-5}$ & 0.02 & $1.46 \times 10^{-4}$ & $6.10 \times 10^{-5} *$ & 0.03 & $9.04 \times 10^{-5}$ & $5.80 \times 10^{-5}$ & 0.06 \\
\hline NDVI (-1-1) & -3.80 & 5.28 & $<0.01$ & -0.87 & 4.17 & $<0.01$ & -5.30 & 6.04 & 0.02 \\
\hline Impervious Surface Area (\%) & $1.86 \times 10^{-2}$ & $3.22 \times 10^{-2}$ & 0.01 & $6.13 \times 10^{-2}$ & $3.13 \times 10^{-2}$ & 0.07 & $-6.35 \times 10^{-2}$ & $6.05 \times 10^{-2}$ & 0.02 \\
\hline Lights at Night (\%) & $-5.96 \times 10^{-2}$ & $5.56 \times 10^{-2}$ & 0.01 & $-8.75 \times 10^{-2}$ & $5.91 \times 10^{-2}$ & 0.02 & $-6.26 \times 10^{-2}$ & $7.33 \times 10^{-2}$ & 0.02 \\
\hline $\mathrm{R} 2$ & & & 0.04 & & & 0.13 & & & 0.12 \\
\hline \multicolumn{10}{|c|}{ Baseline (2000) Urban Characteristics } \\
\hline Area (sq km) & $-1.28 \times 10^{-2}$ & $3.70 \mathrm{x}^{-3 * *}$ & 0.03 & $1.79 \times 10^{-3}$ & $2.29 \times 10^{-3}$ & 0.01 & $-3.69 \times 10^{-4}$ & $6.84 \times 10^{-4}$ & $<0.01$ \\
\hline Population (1000s) & $3.00 \times 10^{-3}$ & $3.81 \times 10^{-3}$ & $<0.01$ & $-5.76 \times 10^{-4}$ & $1.12 \times 10^{-3}$ & 0.01 & $8.94 \times 10^{-4}$ & $2.08 \times 10^{-3}$ & $<0.01$ \\
\hline NDVI (-1-1) & $-2.62 \times 10^{2}$ & $81.48 * *$ & $0<0.01$ & -2.22 & 84.63 & 0.02 & $-1.06 \times 10^{-2}$ & $1.62 \times 10^{-2}$ & 0.07 \\
\hline Impervious Surface Area (\%) & -0.15 & $2.61 \times 10^{-2} * * *$ & 0.06 & $-4.42 \times 10^{-2}$ & $2.32 \times 10^{-2}$ & $<0.01$ & $-9.47 \times 10^{-2}$ & $3.56 \times 10^{-2} * *$ & 0.02 \\
\hline Lights at Night (\%) & $7.03 \times 10^{-2}$ & $2.53 \times 10^{-2} * *$ & 0.05 & $4.04 \times 10^{-2}$ & $2.55 \times 10^{-2}$ & 0.01 & $8.87 \times 10^{-2}$ & $4.69 \times 10^{-2}$ & 0.01 \\
\hline $\mathrm{R} 2$ & & & 0.14 & & & 0.04 & & & 0.10 \\
\hline \multicolumn{10}{|l|}{ Adjustment Factors } \\
\hline Distance to Coast (km) & $-2.56 \times 10^{-3}$ & $4.81 \times 10^{-4 * * *}$ & 0.04 & $-1.71 \times 10^{-3}$ & $3.73 \times 10^{-4} * * *$ & 0.04 & $-1.72 \times 10^{-3}$ & $6.38 \times 10^{-4 * *}$ & 0.02 \\
\hline Annual Rainfall (mm) & $-4.96 \times 10^{-2}$ & $9.82 \times 10^{-3} * * *$ & 0.07 & $-3.76 \times 10^{-2}$ & $8.94 \times 10^{-3 * * *}$ & 0.05 & $-3.70 \times 10^{-2}$ & $1.68 \times 10^{-2} *$ & 0.03 \\
\hline Annual Temperature $\left({ }^{\circ} \mathrm{C}\right)$ & $-3.14 \times 10^{-2}$ & $1.03 \times 10^{-2} * *$ & 0.02 & $-1.19 \times 10^{-2}$ & $1.03 \times 10^{-2}$ & $<0.01$ & $-4.30 \times 10^{-2}$ & $1.67 \times 10^{-2} *$ & 0.02 \\
\hline R2 & & & 0.12 & & & 0.09 & & & 0.07 \\
\hline Full Model R2 & & & 0.51 & & & 0.49 & & & 0.75 \\
\hline
\end{tabular}

${ }^{*}$ p-value $<0.05, * *$ p-value $<0.01, * * *$ p-value $<0.001$ 
Table S4. Multivariate associations between $\mathrm{PM}_{2.5}$ change and urban expansion and urban characteristics - full model.

\begin{tabular}{|c|c|c|c|c|c|c|c|c|c|}
\hline & \multicolumn{9}{|c|}{ Urban Size } \\
\hline & \multicolumn{3}{|c|}{$100,000-250,000(n=235)$} & \multicolumn{3}{|c|}{$250,000-1,000,000(n=340)$} & \multicolumn{3}{|c|}{$>1,000,000(n=255)$} \\
\hline & Coefficient & Std Error & $\mathbf{R 2}$ & Coefficient & Std Error & R2 & Coefficient & Std Error & $\mathbf{R 2}$ \\
\hline \multicolumn{10}{|l|}{ Urban Expansion } \\
\hline Area (sq km) & $2.33 \times 10^{-4}$ & $4.29 \times 10^{-2}$ & $<0.01$ & $3.16 \times 10^{-2}$ & $1.65 \times 10^{-2}$ & 0.02 & $1.09 \times 10^{-3}$ & $1.22 \times 10^{-3}$ & $<0.01$ \\
\hline Population (1000s) & $2.90 \times 10^{-2}$ & $1.90 \times 10^{-2}$ & 0.10 & $-2.81 \times 10^{-3}$ & $5.52 \times 10^{-3}$ & 0.09 & $-5.85 \times 10^{-7}$ & $2.94 \times 10^{-5}$ & 0.01 \\
\hline $\mathrm{R} 2$ & & & 0.11 & & & 0.10 & & & 0.02 \\
\hline \multicolumn{10}{|c|}{ Change in Urban Characteristics } \\
\hline Compactness Index (0-1) & -9.03 & 10.81 & $<0.01$ & -3.36 & 10.40 & $<0.01$ & 21.80 & 12.53 & 0.01 \\
\hline Population Density & $-9.30 \times 10^{-4}$ & $8.57 \times 10^{-4}$ & $<0.01$ & $4.17 \times 10^{-4}$ & $3.44 \times 10^{-4}$ & $<0.01$ & $-3.62 \times 10^{-6}$ & $1.88 \times 10^{-5}$ & 0.02 \\
\hline NDVI (-1-1) & -18.28 & 9.545 & 0.04 & -1.265 & 7.790 & $<0.01$ & 6.21 & 11.15 & 0.02 \\
\hline Impervious Surface Area (\%) & $-0.45^{*}$ & 0.22 & $<0.01$ & -0.17 & 0.15 & 0.01 & -0.40 & 0.23 & $<0.01$ \\
\hline Lights at Night (\%) & $0.4 .0^{*}$ & 0.16 & 0.08 & 0.19 & 0.19 & 0.09 & 0.88 & $0.29 * *$ & 0.22 \\
\hline $\mathrm{R} 2$ & & & 0.13 & & & 0.10 & & & 0.27 \\
\hline \multicolumn{10}{|c|}{ Change in Urban Characteristic Heterogeneity } \\
\hline Population Density & $1.19 \times 10^{-4}$ & $2.83 \times 10^{-4}$ & $<0.01$ & $1.41 \times 10^{-3} * * *$ & $3.40 \times 10^{-4}$ & 0.05 & $4.54 \times 10^{-4}$ & $3.04 \times 10^{-4}$ & 0.05 \\
\hline NDVI (-1-1) & -48.26 & 28.75 & 0.01 & 5.46 & 23.22 & $<0.01$ & -27.86 & 31.59 & 0.03 \\
\hline Impervious Surface Area (\%) & 0.13 & 0.18 & $<0.01$ & $0.37 *$ & 0.17 & 0.04 & -0.15 & 0.32 & $<0.01$ \\
\hline Lights at Night (\%) & -0.2 .8 & 0.31 & $<0.01$ & -0.42 & 0.33 & $<0.01$ & 0.31 & 0.39 & $<0.01$ \\
\hline R2 & & & 0.01 & & & 0.10 & & & 0.08 \\
\hline \multicolumn{10}{|c|}{ Baseline (2000) Urban Characteristics } \\
\hline Area (sq km) & $-4.03 \times 10^{-3}$ & $2.35 \times 10^{-2}$ & $<0.01$ & $1.33 \times 10^{-2}$ & $1.27 \times 10^{-2}$ & 0.01 & $5.55 \times 10^{-3}$ & $3.58 \times 10^{-3}$ & 0.02 \\
\hline Population (1000s) & $-1.57 \times 10^{-2}$ & $2.076 \times 10^{-2}$ & 0.01 & $-8.38 \times 10^{-3}$ & $6.22 \times 10^{-3}$ & 0.01 & $-8.26 \times 10^{-7}$ & $1.09 \times 10^{-6}$ & $<0.01$ \\
\hline NDVI (-1-1) & $1.31 \times 10^{3}$ & $4.44 \times 10^{2} *$ & 0.01 & $-1.13 \times 10^{2}$ & $4.71 \times 10^{2}$ & $<0.01$ & $7.60 \times 10^{2}$ & $8.48 \times 10^{2}$ & 0.05 \\
\hline Impervious Surface Area (\%) & -0.40 & $1.420 \times 10^{-1} *$ & 0.04 & $4.13 \times 10^{-2}$ & 0.13 & $<0.01$ & -0.19 & 0.19 & $<0.01$ \\
\hline Lights at Night (\%) & $4.16 \times 10^{-2}$ & $1.375 \times 10^{-1}$ & $<0.01$ & $-0.28 * * *$ & 0.14 & 0.01 & 0.35 & 0.25 & 0.01 \\
\hline $\mathrm{R} 2$ & & & 0.06 & & & 0.04 & & & 0.08 \\
\hline \multicolumn{10}{|l|}{ Adjustment Factors } \\
\hline Distance to Coast (km) & $-6.48 \times 10^{-3}$ & $2.62 \times 10^{-3} *$ & 0.01 & $-7.81 \times 10^{-3 *}$ & $2.07 \times 10^{-3}$ & 0.05 & $-9.03 \times 10^{-3}$ & $3.34 \times 10^{-3} * *$ & 0.04 \\
\hline Annual Rainfall (mm) & -0.14 & $5.35 \times 10^{-2} *$ & 0.02 & $-4.15 \times 10^{-2}$ & $4.97 \times 10^{-2}$ & $<0.01$ & -0.28 & $8.77 \times 10^{-2} * *$ & 0.08 \\
\hline Annual Temperature $\left({ }^{\circ} \mathrm{C}\right)$ & -0.11 & $5.59 \times 10^{-2} *$ & 0.01 & $-6.36 \times 10^{-2}$ & $5.75 \times 10^{-2}$ & $<0.01$ & -0.17 & $8.76 \times 10^{-2}$ & 0.02 \\
\hline $\mathrm{R} 2$ & & & 0.05 & & & 0.06 & & & 0.14 \\
\hline Full Model R2 & & & 0.36 & & & 0.39 & & & 0.59 \\
\hline
\end{tabular}

*p-value $<0.05$, ** p-value $<0.01$, *** p-value $<0.001$ 


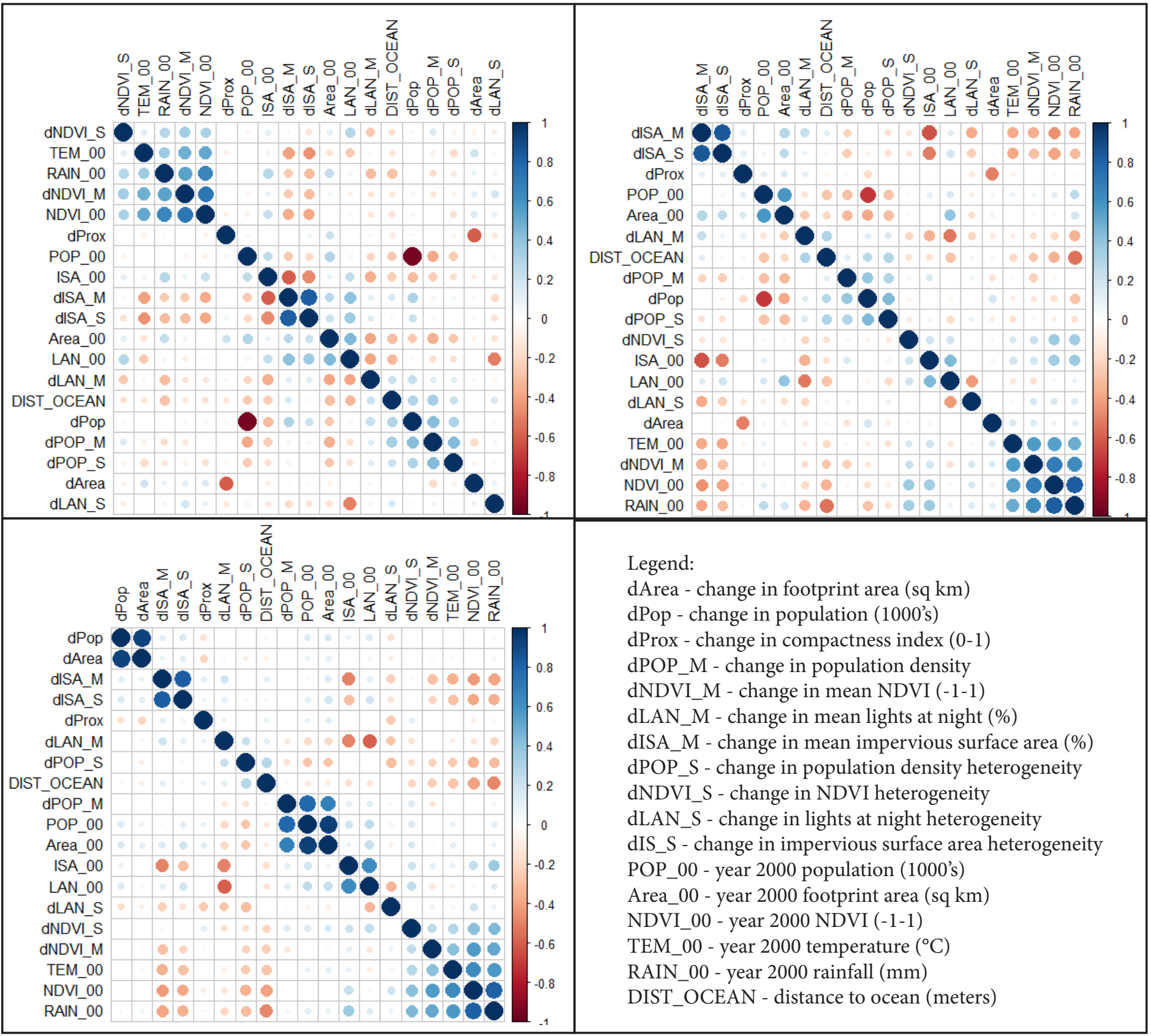

Figure S1. Covariate correlation plots for small (top left), medium, (top right), and large (bottom left) urban areas in East Asia. Variables are organized by hierarchical clustering ("hclust", R corrplot package). Year 2000 NDVI, temperature, rainfall, and change in mean NDVI are strongly positively correlated for all city sizes, as are change in mean impervious surface area and change in surface area heterogeneity. The majority of remaining covariate correlations are either similar between small and medium sized cities (e.g. negative correlation between year 2000 population and change in population density) or between medium and large cities (e.g. positive correlation between year 2000 population and year 2000 area), but are not shared across all city sizes, demonstrating changes in urban typologies as urban footprints grow. 


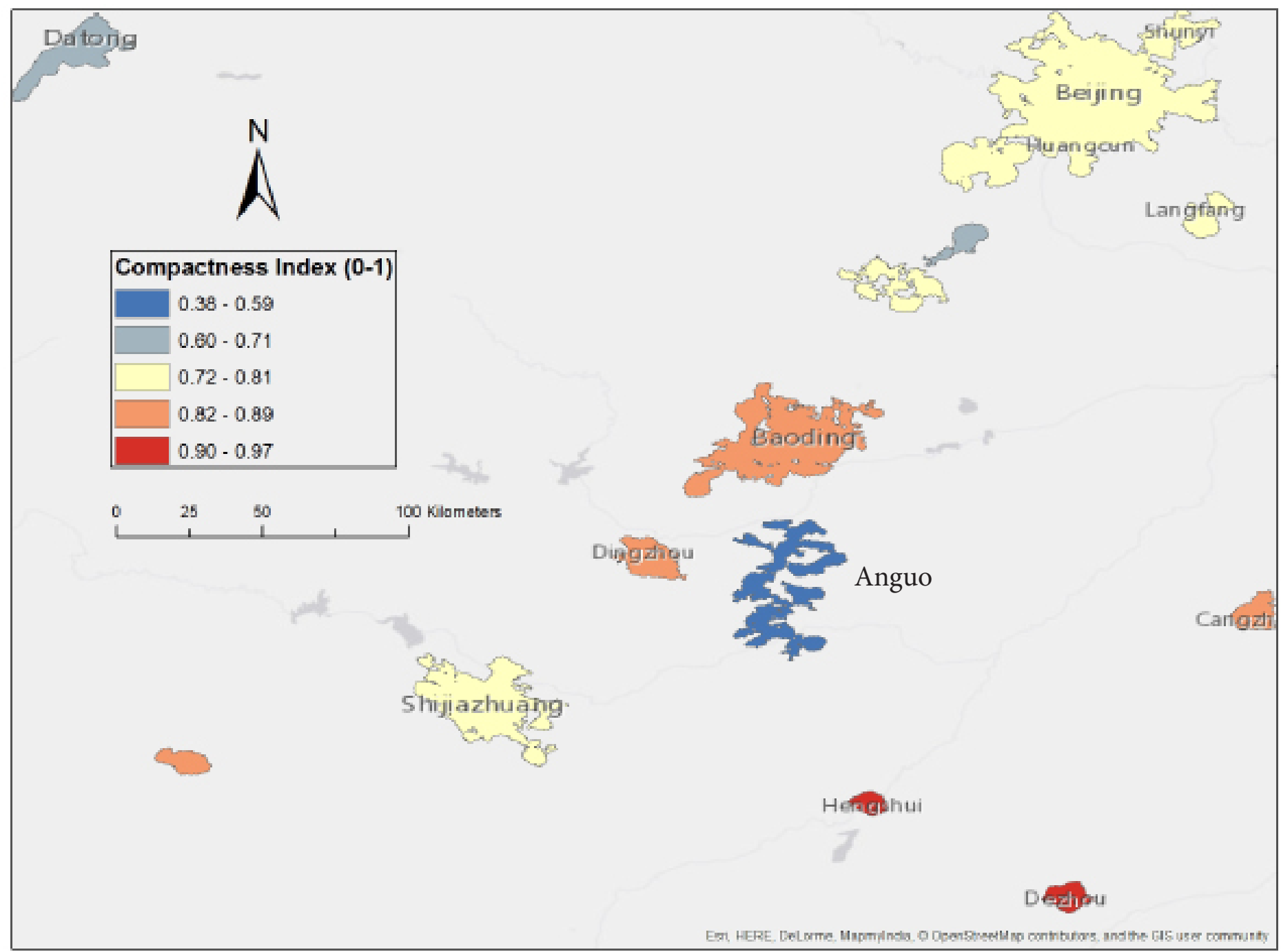

Figure S2. Examples of Compactness Index variation in China. Compactness Index is a measure of the urban sprawl or extent to which a city encroaches on neighboring unurbanized land. Urban areas with a high compactness scores (e.g. Dehzou, China, colored in red in the bottom right) are spherically shaped and tightly centered around the city centroid. Urban areas with low compactness scores (e.g. Anguo, China, colored in blue in the center) have extensive urban sprawl, with a poorly defined city centroid. 


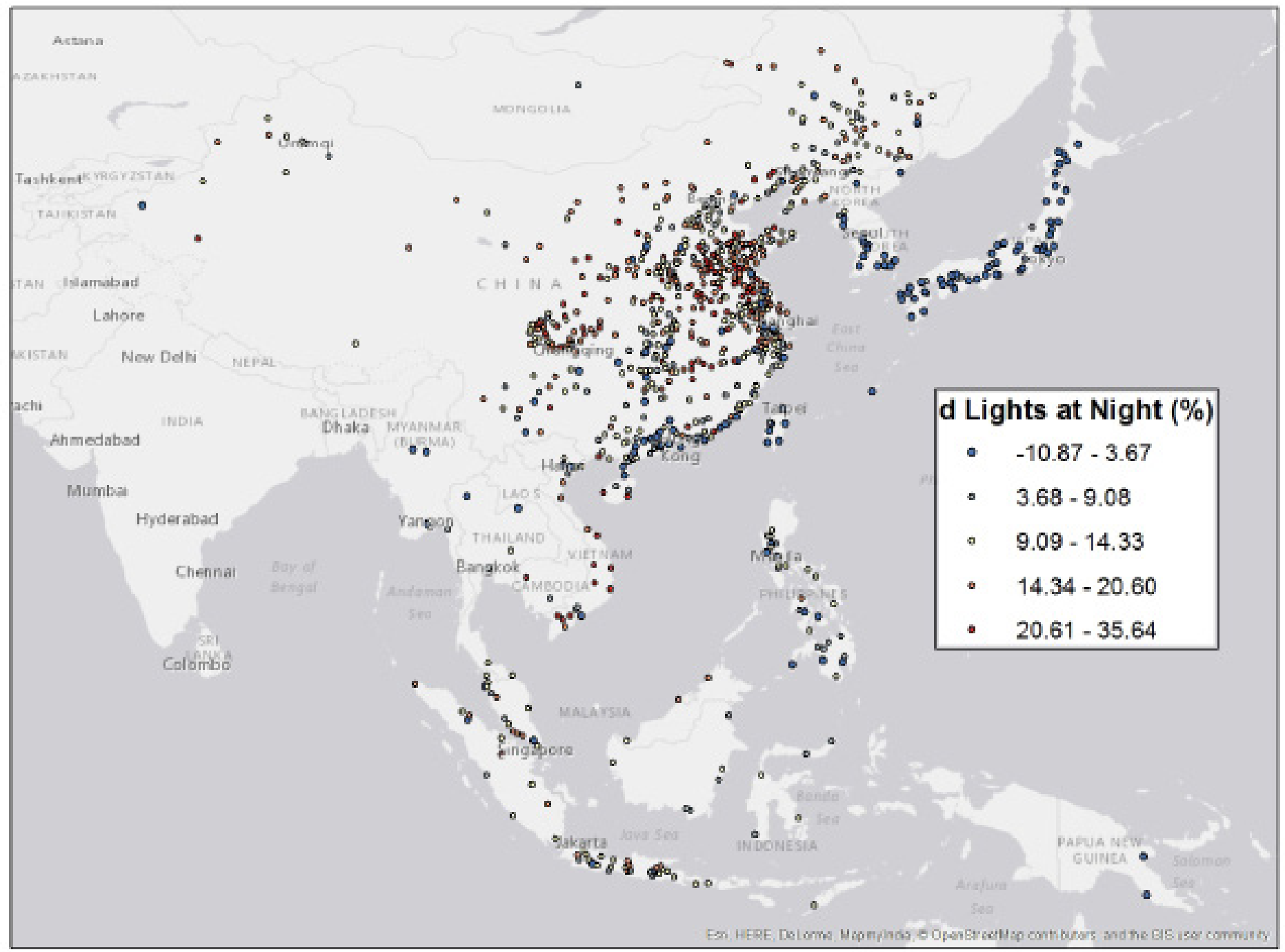

Figure S3. Change in Percent Lights at Night from 2000 - 2010, grouped by quintile, for 830 Cities in Eastern Asia. Reductions were greatest in Japan, South Korea, and Hong Kong, while increases were greatest in Northeastern China. 


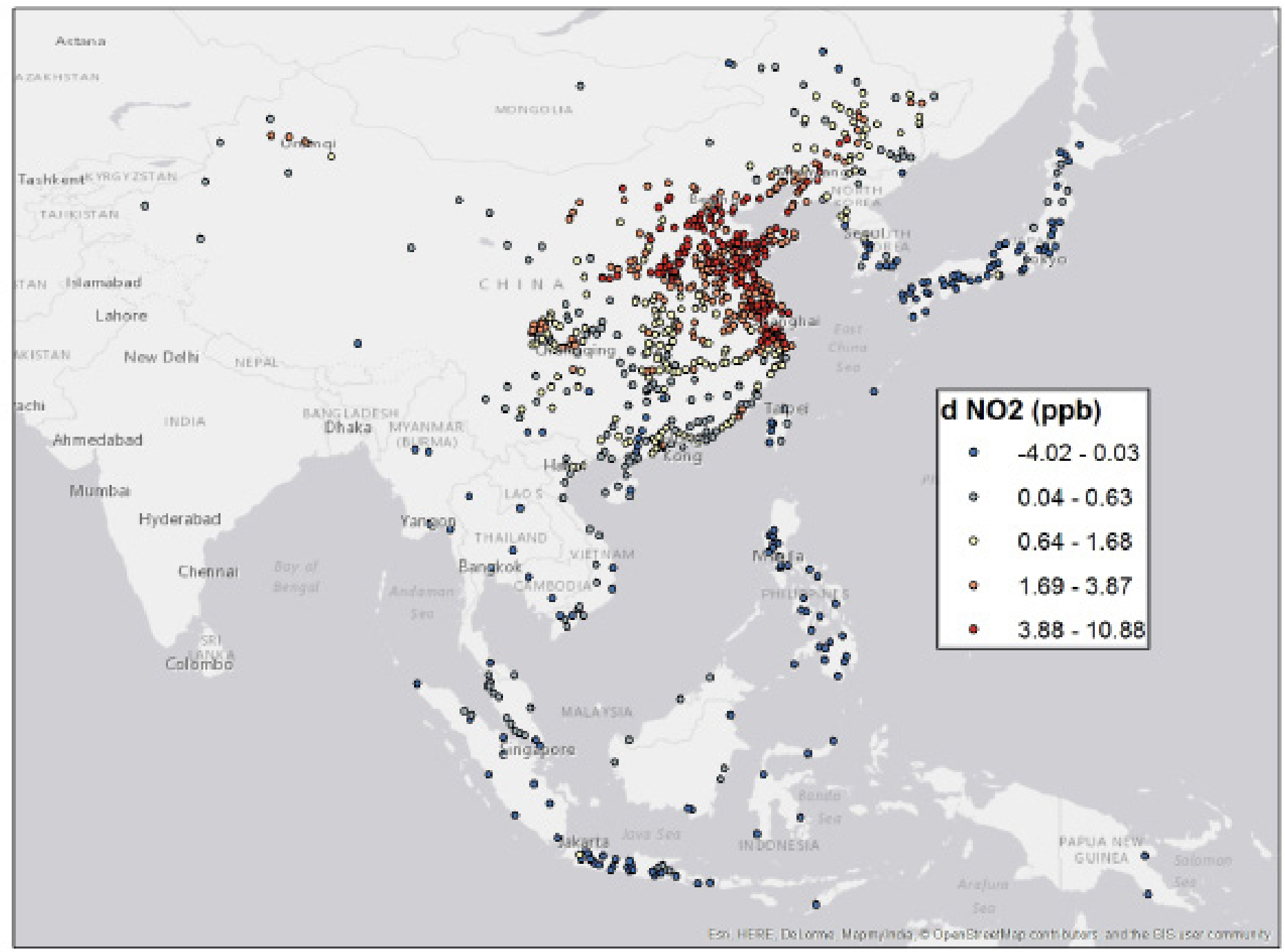

Figure S4. Change in NO2 from 2000 - 2010, grouped by quintile, for 830 Cities in Eastern Asia. Reductions were greatest in Japan, the Philippines, and Singapore, while increases were greatest in Northeastern China. 


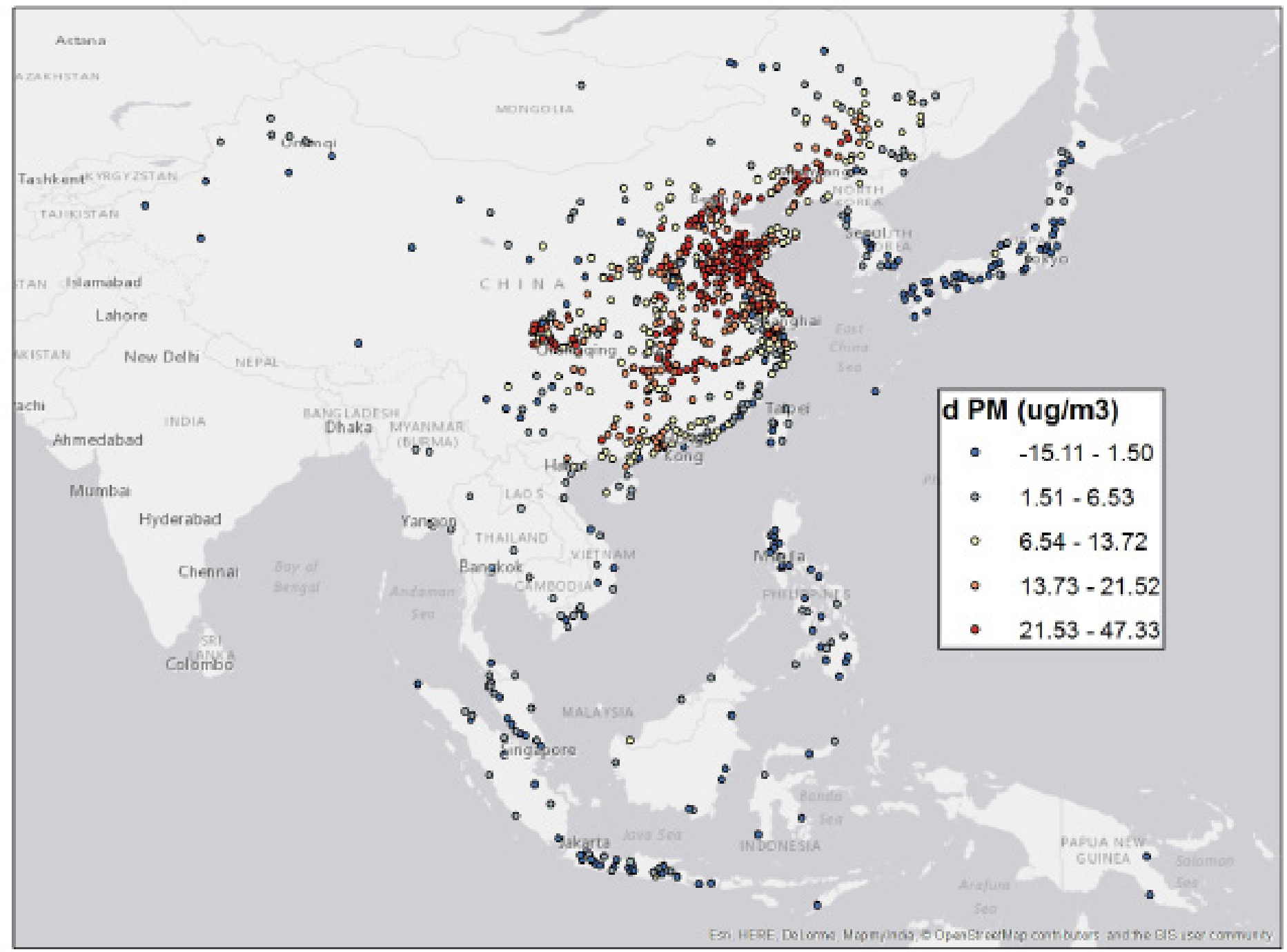

Figure S5. Change in PM2.5 from 2000 - 2010, Grouped by Quintile, for 830 Cities in Eastern Asia. Reductions were greatest in Japan, South Korea, and Hong Kong, while increases were greatest in Northeastern and Central China. 


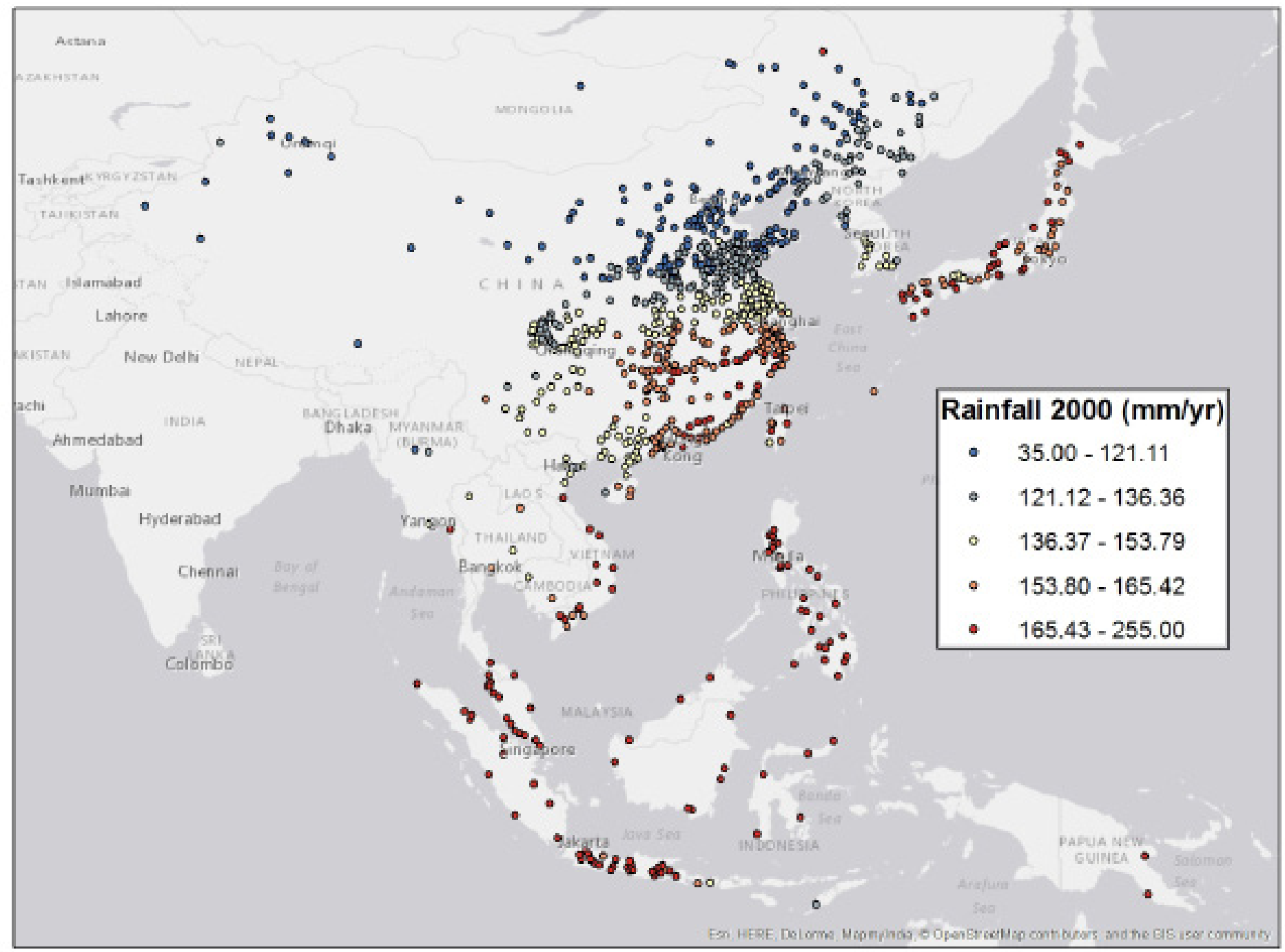

Figure S6. Year 2010 Average Annual Rainfall, Grouped by Quintile, for 830 Cities in Eastern Asia. Rainfall levels are spatially clustered. 